\title{
Comparación de dos dispositivos de medición de potencia y trabajo durante ejercicio defuerza con tecnología inercial flywheel \\ Comparison of two power and work data acquisition systems during resistance exercise employing flywheel inertial technology \\ Luis Manuel Martínez Aranda, Rodrigo Fernández Gonzalo \\ Karolinska Institutet (Suecia)
}

\begin{abstract}
Resumen. El objetivo del presente estudio fue comparar los valores de potencia y trabajo obtenidos en tiempo real mediante los sistemas BlueBrain ${ }^{\mathrm{TM}}$ y SmartCoach ${ }^{\mathrm{TM}}$, durante ejercicio de fuerza en una prensa de piernas equipada con tecnología inercial flywheel, usando diferentes inercias (cargas). Se realizaron ocho series de siete repeticiones a intensidades variable y máxima, con dos minutos de descanso entre series, para cada inercia utilizada en el protocolo: 0.0125; 0.025; 0.0375; 0.05; 0.0625 y $0.075 \mathrm{~kg}^{*} \mathrm{~m}^{2}$, midiendo simultáneamente la potencia media concéntrica y el trabajo total (concéntrico-excéntrico) con ambos dispositivos. Los datos fueron analizados mediante un análisis de regresión lineal y un estudio de correlación. Se observó una correlación altamente significativa en los datos de potencia media entre ambos dispositivos en todas las inercias e intensidades evaluadas, tanto a nivel individual como global, ( $r=.968$; Sig.(bilateral)=.000; $R^{2}=.937$ ). De la misma forma, los datos de trabajo correlacionaron de forma muy significativa ( $r=.978$; Sig.(bilateral)=.000; $R^{2}=.957$ ). Las diferencias de registro entre ambos dispositivos oscilaron en un rango de $2.6-4.3$ \%. Dado el alto grado de significación en las correlaciones analizadas para potencia y trabajo, todo parece indicar que ambos dispositivos se muestran como instrumentos válidos y similares para estimar la potencia y el trabajo durante ejercicios de fuerza con tecnología inercial, siendo de gran ayuda para el seguimiento y control del entrenamiento usando esta tecnología.
\end{abstract}

Palabras clave. BlueBrain, SmartCoach, potencia, flywheel, entrenamiento de fuerza.

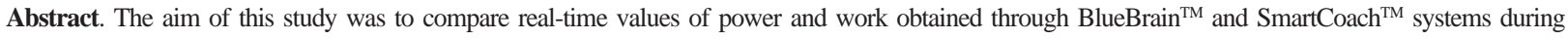
flywheel leg press exercise using different inertias (loads). Eight sets of seven repetitions were performed at variable and maximum intensity for each inertia used (i.e. 0.0125; 0.025; 0.0375; 0.05; 0.0625 and $0.075 \mathrm{~kg}^{*} \mathrm{~m}^{2}$ ), with 2 minutes recovery between sets. Average power during concentric actions and total work (concentric-eccentric) were measured simultaneously using both systems. Data were analysed using a linear regression analysis and correlation procedures. Strong significant correlations were observed in average power results between both data acquisition systems for all individual inertias and intensities evaluated, as well as for overall data ( $\mathrm{r}=.968$; Sig.(2-tailed)=.000; $\mathrm{R}^{2}=.937$ ). In addition, work values showed clear significant correlations ( $\mathrm{r}=.978$; Sig.(2-tailed) $=.000$; $\left.\mathrm{R}^{2}=.957\right)$. Differences between devices oscillated over a range of $2.6-4.3 \%$. The strong correlations found in power and work values seem to indicate that both data acquisition systems are similar and valid to estimate power and work during resistance exercises employing flywheel inertial technology. Thus, both devices may represent a helpful tool to control and follow up training using flywheel technology.

Keywords. BlueBrain, SmartCoach, power, flywheel, resistance training.

\section{Introducción}

La potencia y fuerza muscular son cualidades esenciales en el ámbito de la preparación deportiva y el desarrollo físico. Un deportista necesita ser capaz de expresar la mayor fuerza necesaria en el menor tiempo posible para las acciones que su disciplina deportiva requiera. Por ello, el entrenamiento destinado a desarrollar valores máximos de fuerza y potencia en determinadas acciones que requieren aceleraciones y cambios rápidos de dirección, puede ser uno de los puntos clave en los programas de mejora del rendimiento deportivo(Ferrer Contreras, 2007). Además, este tipo de entrenamiento tiene implicaciones directas en la mejora significativa de la fuerza y componentes de la velocidad(Cronin \& Hansen, 2005; Loturco, Artioli, Kobal, Gil \& Franchini, 2014; Ratamess, 2012), así como de mejora en la fuerza muscular y calidad de vida en poblaciones especiales (Torres Luque, García-Martos, Villaverde Gutiérrez \& Garatachea Vallejo, 2010; Víquez Ulate \& Mora Campos, 2011). En este sentido, el entrenamiento de fuerza con tecnología inercial es una metodología excelente para mejorar la potencia e incrementar la masa muscular, ya que ofrece resistencia variable e ilimitada tanto en la acción muscular concéntrica(CON) como excéntrica (EXC), gracias a la inercia generada por un volante rotatorio, contrastando de esta manera con el entrenamiento tradicional basado en carga constante (Niewiadomski, Laskowska, G1'siorowska, Cybulski, Strasz, \& Langfort, 2008). De hecho, el uso de esta tecnología en el entrenamiento proporciona un estímulo en el músculo de mayor calidad con respecto al entrenamiento tradicional, mostrando mayores adaptaciones de fuerza, masa muscular, potencia y activación neuronal que el entrenamiento de fuerza convencional (Norrbrand, Fluckey, Pozzo \& Tesch, 2008; Norrbrand, Pozzo \& Tesch, 2010; Onambele, Maganaris, Mian, Tam,

Fecha recepción: 07-07-15- Fecha envío revisores: 07-07-15- Fecha de aceptación: 17-12-15 Luis Manuel Martínez Arand m82maarl@uco.es
Rejc, McEwan \& Narici, 2008), posiblemente debido a la sobrecarga excéntrica que proporciona (Fernandez-Gonzalo, Nissemark, Aslund, Tesch \& Sojka, 2014).

La tecnología flywheel cuenta con la posibilidad de trabajar con diferentes inercias de entrenamiento y además ha mostrado ser un sistema bastante fiable y seguro. Por ello, ha sido utilizado con diferentes objetivos, no sólo por multitud de equipos deportivos y atletas de alto nivel para la mejora del rendimiento (Cuenca-Fernández, LópezContreras \& Arellano, 2015), sino también para la prevención de lesiones (Askling, Karlsson \& Thorstensson, 2003; De Hoyo, Pozzo, Sañudo, Carrasco, Gonzalo-Skok, Domínguez-Cobo \& Morán-Camacho, 2015), o en el ámbito de la rehabilitación de enfermedades que conllevan un deterioro muscular importante (Fernandez-Gonzalo et al., 2014).

Por otro lado, la medición y registro de la potencia y la ratio potencia-carga pueden ser herramientas interesantes para optimizar el entrenamiento y evaluar la fuerza en función de los objetivos y criterios de especificidad deseados (Smilios, Sotiropoulos, Christou, Douda, Spaias, \& Tokmakidis, 2013). Uno de los elementos disponibles para ello, cuyo uso está muy extendido, es el encoder, el cual permite obtener una gran cantidad de datos de variables como la fuerza, la velocidad, la potencia y el trabajo en cada repetición y por consiguiente las relaciones que se pueden obtener entre ellas. Además estos dispositivos cuentan con un excelente grado de fiabilidad y precisión con un error de estimación bajo.

Son muchos los estudios que han usado diferentes encoders para medir la producción de potencia, trabajo, velocidad y otras variables durante multitud de ejercicios de fuerza diferentes (González- Badillo \& Sánchez-Medina, 2010; González-Badillo, Pareja-Blanco, RodríguezRosell,Abad-Herencia, Del Ojo-López, \& Sánchez-Medina, 2015). Sin embargo, también se han utilizado para otros muchos objetivos, como por ejemplo, la validación de su uso en un entrenamiento específico (Drinkwater, Galna, McKenna, Hunt \& Pyne, 2007), validaciones en test relacionados con la salud en mayores (Lindemann, Farahmand, Klenk, Blatzonis \& Becker, 2015), o la comparación entre diversos 
modelos o métodos de medición de variables (Hilmersson, Edvardsson \& Tornberg, 2015; Squadrone, Rodano \& Preatoni, 2012).

Entre toda la gama de enconders disponibles encontramos SmartCoach ${ }^{\mathrm{TM}}$, utilizado en gran cantidad de estudios científicos y concretamente de forma reciente en trabajos con tecnología inercial flywheel (Fernandez-Gonzalo, Lundberg, Alvarez-Alvarez \& De Paz, 2014; Lundberg, Fernandez-Gonzalo, Gustafsson \& Tesch, 2013). Por otro lado, seha desarrollado recientemente un dispositivo portátil einalámbrico, BlueBrain $^{\mathrm{TM}}$, diseñado específicamente para la evaluación y análisis de la potencia muscular y el trabajo realizado en maquinaria equipada con tecnología inercial nHance ${ }^{\mathrm{TM}}$. Sin embargo, no existe hasta la fecha ningún estudio publicado que utilice el dispositivo BlueBrain ${ }^{\mathrm{TM}}$ en sistemas con tecnología inercial flywheel, para los que específicamente se ha creado. Por extensión, tampoco hay constancia de estudios que comparen ambos dispositivos.

Por lo tanto, el objetivo de este estudio piloto ha sido comparar los valores de potencia y trabajo obtenidos en tiempo real mediante los sistemas BlueBrain ${ }^{\mathrm{TM}}$ y SmartCoach ${ }^{\mathrm{TM}}$, durante un ejercicio de prensa de piernas con tecnología inercial flywheel usando diferentes inercias (cargas). La hipótesis fue que para el registro de dichas variables, ambos dispositivos son consistentes cuando se utilizan al unísono en la valoración del ejercicio especificado.

\section{Material y método}

\section{Participantes}

Para la realización de este estudio piloto se eligió a dos sujetos sanos y moderadamente entrenados de entre ocho voluntarios que se evaluaron para la realización de los test. Se exigióno haber tenido lesión muscular u ósea al menos en los seis meses previos al estudio y no realizar actividad extenuante además de ejercicios de fuerza para las extremidades inferiores al menos durante las 48 horas previas a cada intervención

La razones de escoger a una muestra final tan pequeña fueron principalmente la similitud de los dos sujetos tanto en características antropométricas ( $31.5 \pm 0.7$ años; $179 \pm 5.6 \mathrm{~cm} ; 79.2 \pm 4.5 \mathrm{~kg}$ ), el estado de forma y tiempo de entrenamiento semanal, con dos-tres días de actividad vigorosa ( $85 \pm 7 \mathrm{~min}$.) y actividad moderada ( $65 \pm 7 \mathrm{~min}$.), pero sobre todo por su experiencia en entrenamiento de fuerza con tecnología inercial, ya que de forma reciente habían participado en un estudio de mayor envergadura usando dicha tecnología en nuestro laboratorio. Este hecho hizo posible que el sesgo de variabilidad por motivos de ejecución técnica se redujera al mínimo.

Toda la información acerca de los propósitos del estudio y los riesgos potenciales asociado a los test fueron puestos en conocimientos de los sujetos, obteniendo el consentimiento informado correspondiente. El protocolo del estudio fue aprobado por el comité ético regional.

\section{Instrumentos}

Todos los test fueron llevados a cabo en una prensa de piernas (Leg Press) equipada con tecnología inercial flywheel (YoYo® Technology Inc., Stockholm, Sweden). Los ajustes de la máquina fueron individualizados para cada sujeto y mantenidos a lo largo de las pruebas realizadas. La cadera y el pecho se fijaron a la máquina con las bandas de ajuste que la misma proporciona. Para la realización de los test se utilizaron ruedas específicas, las cuales proporcionaron seis inercias diferentes $\left(0.0125,0.025,0.0375,0.05,0.0625\right.$ y $\left.0.075 \mathrm{~kg}^{*} \mathrm{~m}^{2}\right)$.

Tanto la potencia media de la fase CON medida en Vatios (W) como el trabajo total realizado en las fases CON y EXC medido en Julios (J), fueron calculados para cada repetición mediante la medición de la velocidad rotacional con la ayuda de dos dispositivos diferentes con su software adjunto. Por un lado, BlueBrain ${ }^{\mathrm{TM}}$ (nHance- Driven by YoYo® Technology Inc., Stockholm, Sweden; BB), que además de aportar datos de potencia y trabajo, ofrece un feedback gráfico en tiempo real y se adapta a cualquier máquina $\mathrm{nHance}^{\mathrm{TM}}$. La ausencia de requerimientos de instalación o mantenimiento, así como su diseño, su facilidad a la hora de exportar los resultados y la actualización del software de forma gratuita, son las ventajas con las que cuenta este novedoso dispositivo. El segundo dispositivo utilizado fue SmartCoach ${ }^{\mathrm{TM}}$ (SmartCoach Europe AB, Stockholm, Sweden; SC), el cual ofrece gran cantidad de datos tanto de potencia y trabajo como de velocidad. Ofrece feedback visual y sonoro en tiempo real y se adapta a cualquier máquina, ya sea de peso libre y en determinadas máquina de tecnología inercial. Ambos dispositivos se perfilan como una herramienta muy completa para análisis del entrenamiento.

\section{Diseñoy Procedimiento}

Este estudio evaluó mediante un test de medidas repetidas, la producción de potencia media CON y el trabajo total CON-EXC durante un ejercicio de fuerza en una prensa de piernas equipada con tecnología inercial usando seis inercias diferentes $(0.0125,0.025,0.0375,0.05$, 0.0625 y $0.075 \mathrm{~kg}^{*} \mathrm{~m}^{2}$ ). Los participantes en el estudio realizaron cuatro series de siete repeticiones con dos minutos de descanso entre series, para cada una de las dos intensidades (variable entre 60-90 \% y máxima al 100\%) y para cada una de las seis inercias establecidas, resultando en un total de 96 series y 672 repeticiones. Tras una repetición previa en torno al $50 \%$ para iniciar el movimiento de la rueda inercial, cada sujeto fue advertido de la necesidad de realizar las repeticiones con una ejecución correcta, realizando la fase $\mathrm{CON}$ desde $\sim 70^{\circ}$ de flexión de rodilla hasta casi la extensión completa $\left(\sim 175^{\circ}\right)$. Posteriormente, al finalizar la fase CON, la correa colocada en el eje rotacional rebobina la rueda debido a la fuerza inercial. A partir de ese momento se pidió a los sujetos que durante el primer tramo de la fase EXC se resistiera suavemente el movimiento, para a continuación aplicar la fuerza necesaria para romper dicha inercia y detener el movimiento aproximadamente a $70^{\circ}$ de flexión de rodilla (en la posición de inicio), para seguidamente volver a realizar una fase CON iniciando con ello una nueva repetición. Este protocolo se ha empleado con éxito para obtener una sobrecarga de fuerza y potencia en la fase EXC del movimiento (Fernandez-Gonzalo, et al., 2014).

Se estableció un período de 4 semanas para la toma de datos, permitiendo con ello un periodo de al menos 48 horas entre cada sesión de test analizada, de acuerdo con Atkinson \& Nevill (1998), quienes en su revisión afirman que los test de rendimiento físico necesitan más de 24 horas de recuperación cuando se trata de pruebas con medidas repetidas o consecutivas.

Previamente a la realización de las pruebas se ejecutaron dos sesiones de familiarización y entrenamiento con la prensa de piernas en las que se establecieron los porcentajes aproximados a los que tendrían que realizar las series variables. En cualquier caso, como ya se ha referido anteriormente, eran sujetos con experiencia en la utilización de esta metodología y habían participado de forma reciente en otro estudio utilizando este tipo de tecnología, por lo que fue relativamente sencillo establecer dichos parámetros. Todas las sesiones fueron precedidas de un calentamiento estandarizado (5 min. en cicloergómetro + extensiones en prensa con cargas muy bajas) y realizadas a la misma hora del día ( \pm 1.5 horas). Se ofreció un feedback continuo sobre los valores de potencia y trabajo en todas las sesiones. Así mismo, se ofreció información de la posición de las piernas en la prensa, flexión de rodilla y la correcta ejecución del ejercicio sólo durante las sesiones de familiarización. Durante las sesiones de test, se intentó que los sujetos se focalizaran al máximo en el correcto desarrollo del protocolo establecido.

\section{Análisis estadístico}

Los datos de este estudio están expresados en estadísticos descriptivos de media, desviación típica y porcentajes. Debido a que las ruedas inerciales y BB tienen exactamente las mismas unidades de inercia y difieren levemente de las inercias mostradas por defecto en el interfaz de SC, se procedió a realizar el ajuste matemático para los valores reportados. Para comparar los datos reportados de las variables potencia y trabajo se llevó a cabo un estudio de correlación y análisis de regresión lineal. También se obtuvo mediante análisis de fiabilidad el ICC (coeficiente de correlación intraclase) en términos absolutos y de consistencia para las dos variables y en las dos intensidades analizadas, así como en 
términos generales con el total de series realizadas. Para el análisis de datos se utilizó el programa estadístico SPSS v.21 (SPSS Inc., Chicago, IL). El nivel de significación establecido fue $p<.05$. La siguiente ecuación fue la usada para estimar los porcentajes de diferencias absolutas entre las series de datos, tomando como referencia los datos de BB: \% Diferencia BB vs. SC= 100-(SC*100/BB)

\section{Resultados}

Los valores de potencia media CON obtenidos con BB y SC fueron $313.9 \pm 91.5$ y $303.5 \pm 84.6 \mathrm{~W}$, respectivamente, en el total de series realizadas a intensidad variable $(n=336) ; 391.6 \pm 66.3$ y $373.3 \pm$ $57.5 \mathrm{~W}$, respectivamente, en el total de series de intensidad máxima ( $\mathrm{n}=336$ ); $352.8 \pm 88.8$ y $338.4 \pm 80.2 \mathrm{~W}$, respectivamente, en el total de series realizadas ( $\mathrm{n}=672$ ). Así mismo, se obtuvieron unos valores totales de trabajo CON-EXC de $670.7 \pm 159.8$ para BB y $651.8 \pm 165.7 \mathrm{~J}$ para SC para series variables; $796.8 \pm 125.0$ (BB) y $774.7 \pm 142.4 \mathrm{~J}$ (SC) para series máximas; por último, $733.8 \pm 156.6$ (BB) y $713.2 \pm$ 166.2 J (SC) para el total de series.

En las series realizadas a intensidad máxima, los valores máximos de potencia media CON se obtuvieron utilizando la inercia más baja(0.0125 $\mathrm{kg}^{*} \mathrm{~m}^{2}$ ), y fueron de $461.7 \pm 59.3 \mathrm{~W}$ para BB y $425.2 \pm 51.5 \mathrm{~W}$ para SC. Los valores mínimos de potencia media CON se obtuvieron en la inercia más alta $\left(0.075 \mathrm{~kg}^{*} \mathrm{~m}^{2}\right)$, y fueron de $312.2 \pm 32.3 \mathrm{~W}$ para BB y 309.7 \pm 34.1 para SC (Figura 1). El comportamiento para la variable trabajo CON-EXC fue inverso, obteniéndose los valores mínimos (595.2 \pm 59.3 J para BB y 532.7 \pm 45.7 J para SC) en la inercia más baja (0.0125 $\mathrm{kg}^{*} \mathrm{~m}^{2}$ ), mientras que los valores máximos fueron de 913.6 $\pm 81.7 \mathrm{~J}$ para BB y $911.2 \pm 65.3$ J para SC usando la segunda inercia más alta 0.0625 $\mathrm{kg}^{*} \mathrm{~m}^{2}$ (Figura 2).

Se encontraron correlaciones significativas $(p<.05)$ entre las variables potencia media CON y trabajo CON-EXC para todas las inercias analizadas en las series realizadas a intensidad variable, máxima y para el total de series realizadas en el estudio. En las figuras 3 y 4 se muestra

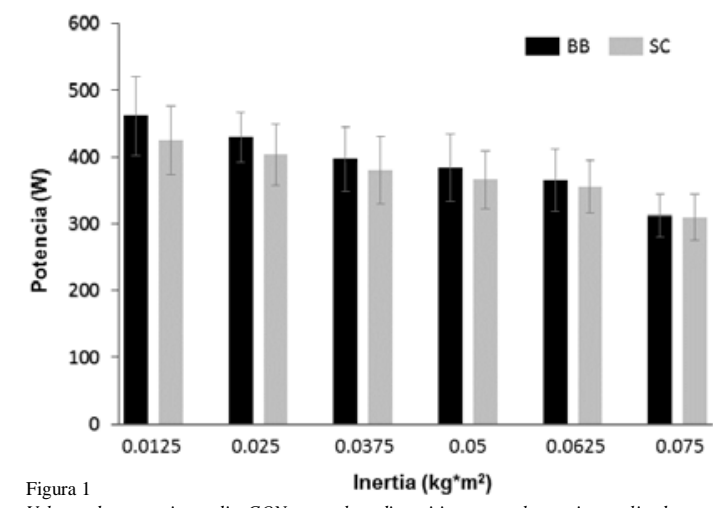

Valores de potencia media CON en ambos dispositivos para las series realizadas a máxima intensidad ( $n=336$ ) en cada una de las inercias analizadas.

CON: Concéntrica; BB: BlueBrain ${ }^{\mathrm{TM}}$; SC: SmartCoach ${ }^{\mathrm{TM}}$

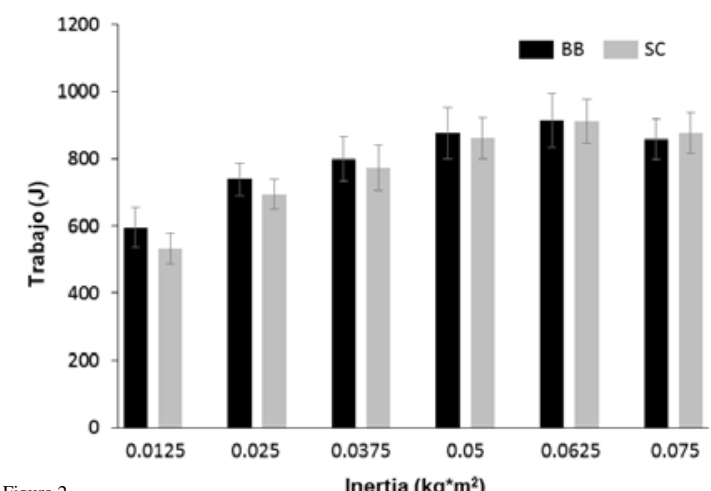

Figura 2 Inertia $\left(\mathbf{k g}^{*} \mathbf{m}^{2}\right)$

Valores de trabajo CON-EXC en ambos dispositivos para las series realizadas a máxima intensidad ( $n=336)$ en cada una de las inercias analizadas.

CON: Concéntrica; EXC: Excéntrica; BB: BlueBrain ${ }^{\mathrm{TM}}$; SC: SmartCoach ${ }^{\mathrm{TM}}$

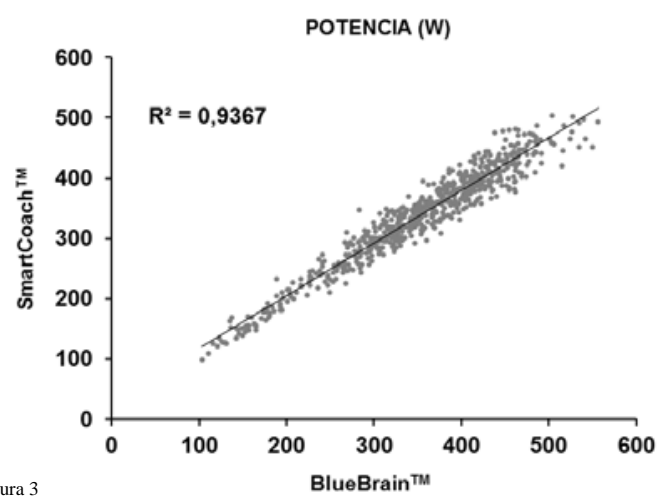

Figura 3

Regresión lineal de la relación entre BlueBrain ${ }^{T M}$ y SmartCor

media CON en el total de series e inercias analizadas $(n=672)$.

CON: Concéntrica; W: Vatios; R²: Coeficiente de Determinación.

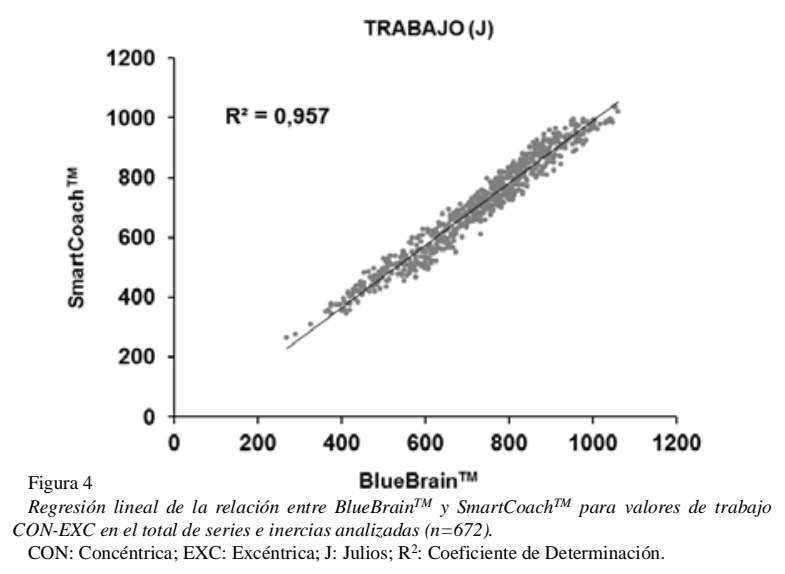

la regresión lineal para potencia y trabajo del total de series analizadas en el estudio.

En la tabla 1 se reflejan todos los valores de coeficiente de correlación de Pearson $(r)$ y coeficiente de determinación $\left(R^{2}\right)$ resultantes desglosados para las variables potencia y trabajo en cada una de las inercias e intensidades evaluadas, así como en la totalidad de series realizadas. Se observa como para la variable potencia se obtuvieron valores de .974-.948 en series variables, .934-.872 en series máximas y .968 - .937 en el total de series para $r$ y $R^{2}$ respectivamente. Para la variable trabajo dichos coeficientes fueron todavía más elevados, con .981 - .962 en series variables, .967 - .940 en series máximas y .978.957 para el total de series.

Los porcentajes absolutos de diferencia entre BB y SC se mantuvieron en un rango muy bajo, de $2.6-4.3 \%$ (Tabla 1). Los ICC resultantes en términos absolutos y de consistencia oscilan entre .949.981 en la variable potencia y $.969-.988$ en la variable trabajo para el total de series analizadas. El índiceAlpha de Cronbach osciló entre.966 -.990 .

\section{Discusión}

El objetivo de este trabajo fue comparar y analizar los valores de potencia media CONy trabajo CON-EXC obtenidos de manera simultánea mediante dos dispositivos (BB-SC), durante ejercicio de fuerza en una prensa de piernas equipada con tecnología inercial flywheel. Para ello, se usaron dos tipos de intensidad (variable y máxima) y seis inercias diferentes. Estees el primer estudio que utiliza el sistema BlueBrain ${ }^{\mathrm{TM}}$, además de realizar una comparativa con otro dispositivo ampliamente utilizado en investigación que también se adaptan al trabajo con tecnología inercial flywheel, como es el caso de SmartCoach ${ }^{\mathrm{TM}}$.

Confirmando nuestra hipótesis inicial, las correlaciones entre ambos dispositivos para los valores de potencia y trabajo en series de intensidad variable y máxima son elevadas, siendo así para cada una de las seis inercias evaluadas. 
Tabla 1

Correlaciones $y \%$ de diferencias entre los dispositivos BlueBrain ${ }^{\mathrm{TM}}$ y SmartCoach ${ }^{\mathrm{TM}}$ para series de intensidad variable, series de intensidad máxima y el total de series realizadas, en cada una de las inercias analizadas.

\begin{tabular}{|c|c|c|c|c|c|c|c|c|c|c|c|c|}
\hline \multirow{3}{*}{$\begin{array}{c}\text { Inercia } \\
\left(\mathrm{kg}^{*} \mathrm{~m}^{2}\right)\end{array}$} & \multicolumn{4}{|c|}{ S. VARIABLES $(\mathrm{n}=336)$} & \multicolumn{4}{|c|}{ S. MÁXIMAS $(\mathrm{n}=336)$} & \multicolumn{4}{|c|}{ T. SERIES $(n=672)$} \\
\hline & \multicolumn{2}{|c|}{ POTENCIA } & \multicolumn{2}{|c|}{ TRABAJO } & \multicolumn{2}{|c|}{ POTENCIA } & \multicolumn{2}{|c|}{ TRABAJO } & \multicolumn{2}{|c|}{ POTENCIA } & \multicolumn{2}{|c|}{ TRABAJO } \\
\hline & $r$ & $R^{2}$ & $r$ & $R^{2}$ & $r$ & $R^{2}$ & $r$ & $R^{2}$ & $r$ & $R^{2}$ & $r$ & $R^{2}$ \\
\hline 0.0125 & $.956 * *$ & .913 & $.970^{* *}$ & .941 & $.924^{* *}$ & .855 & $.936^{* *}$ & .877 & $.966 * *$ & .933 & $.971^{* *}$ & .944 \\
\hline 0.025 & $.945 * *$ & .892 & $.914 * *$ & .835 & $.837 * *$ & .802 & $.863^{* *}$ & .845 & $.917 * *$ & 841 & $.922 * *$ & .851 \\
\hline 0.0375 & $.955 * *$ & .913 & $.979 * *$ & .958 & $.890^{* * *}$ & .793 & $.933 * *$ & .870 & $.942 * *$ & .888 & $.972 * *$ & .945 \\
\hline 0.05 & $.929^{* *}$ & .863 & $.953 * *$ & .909 & $.905^{* *}$ & .819 & $.903 * *$ & .816 & $.928 * *$ & .861 & $.942 * *$ & 888 \\
\hline 0.0625 & $.986 * *$ & .973 & $.992 * *$ & .984 & $.932^{* *}$ & .869 & $.956 * *$ & .914 & $.983 * *$ & 967 & $.990^{* *}$ & .981 \\
\hline 0.075 & $.972^{* *}$ & .944 & $.988^{* *}$ & .977 & $.887 * *$ & .787 & $.927^{* *}$ & .859 & $.973 * *$ & 948 & $.988^{* *}$ & .977 \\
\hline TOTAL & $.974^{* *}$ & .948 & $.981 * *$ & .962 & 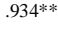 & .872 & $.967^{* *}$ & .940 & $.968^{* *}$ & .937 & $.978^{* *}$ & .957 \\
\hline $\begin{array}{l}\text { \% Diferencia } \\
\text { BB vs.SC }\end{array}$ & & & & & & & & & & & & \\
\hline
\end{tabular}

determinación; **La correlación es significativa al nivel 0.01 (bilateral).

En este sentido, estudios previos donde se ha evaluado la potencia en diversos ejercicios de fuerza utilizando tecnología inercial, se han empleado inercias (cargas) que oscilan entre $0.11 \mathrm{~kg}^{*} \mathrm{~m}^{2}$ (Lundberg, Fernandez-Gonzalo \& Tesch, 2014) y 0.036 kg*m² (Fernandez-Gonzalo, et al., 2014). Este último trabajo se llevó a cabo en una prensa de piernas con tecnología inercial y con una única inercia, con un protocolo similar a nuestro estudio en cuanto a número de series y repeticiones se refiere, pero con un tiempo de recuperación superior (3 minutos). Respecto a ello, en un reciente estudio, Hernandez Davó et al. (2015), no observaron diferencias significativas entre 2 y 3 minutos como tiempo de recuperación, para series de potencia muscular y en referencia a posibles descensos en sus niveles.

El presente estudio ha utilizado una gama más amplia de cargas inerciales, posibilitando de esta manera ver el comportamiento en el registro de ambos dispositivos ante los incrementos y descensos de resistencia inercial, así como de la velocidad en la ejecución del ejercicio a través de las diferentes inercias. Como se observa en las figuras 1 y 2 , durante las series máximas los valores de potencia media CON han ido descendiendo de forma paulatina a medida que se aumentaba la inercia. Además, se observa como el porcentaje de diferencia de registro entre ambos dispositivos va también disminuyendo con el aumento de carga inercial. Con un comportamiento inverso encontramos los valores de trabajo CON-EXC, los cuales van aumentando conforme se aumenta de inercia, así mismo y al igual que con los valores de potencia, al aumentar la carga se reducen las diferencias entre BB y SC.

La comparación con estudios previos es bastante compleja por diferencias metodológicas, por ser ejercicios específicos con tecnología inercial y la utilización de mayor número de inercias, así como por la novedad que supone BB y esta comparativa con SC. Aun así, Drinkwater et al. (2007) con un protocolo parecido (cuatro series de seis repeticiones con dos minutos y 45 segundos de descanso) en ejercicios de fuerza con medición por encoder, muestra como los valores de potencia media CON disminuyen de forma importante, a través de las series realizadas en función de variaciones ascendentes en la carga. Este hecho respalda lo observado en nuestro estudio, donde hubo un comportamiento similar al referirnos a los valores de potencia media CON en series máximas.

Como se puede observar en las figuras 3, 4 y en la tabla 1, el estudio de correlación y el análisis de regresión lineal aportan datos interesantes que demuestran que ambos dispositivos registran de forma muy pareja los valores referentes a potencia media CON y trabajo CON-EXC. Sin embargo, se aprecia como las series máximas no solo muestran coeficientes de Pearson y determinación más bajos que en las series variables, sino que también muestran un porcentaje de diferencia entre BB y SC más alto, concretamente en los valores de potencia. Estas diferencias absolutas de porcentajes entre ambos dispositivos podrían ser lógicas debido a que los dos sistemas se basan en un modelo matemático válido, pero algo diferente en cuanto a la configuración de las inercias en la interfaz, para lo cual se necesitó realizar un ajuste de estimación. En este sentido, estudios previos presentaban la misma circunstancias en sus comparativas (Hilmersson, et al., 2015; Squadrone, et al., 2012). A pesar de ello, en líneas generales las diferencias entre ambos dispositivos es bastante pequeña (no supera el $4.3 \%$ de media), siendo de un $2.6 \%$ de media para las series en las que hubo mayor variabilidad en la inten- sidad de ejecución y por lo tanto también en la velocidad de ejecución. En este sentido como se aprecia en las figuras 1 y 2, parece que la velocidad de ejecución podría jugar un papel importante, no solo en valores decrecientes de producción de potencia, sino también en el porcentaje de diferencia de valores registrados por ambos dispositivos.

Squadrone et al. (2012), realizaron un estudio comparativo sobre producción de velocidad y potencia durante un ejercicio de fuerza medido con dos dispositivos diferentes. Encontraron unos porcentajes absolutos de diferencia en la medición de ambos de $3.8 \pm 2 \%$ para los valores de potencia media, no encontrando diferencias significativas en este sentido y concluyendo que el límite de error era razonable y las mediciones consistentes. Estos hallazgos refuerzan nuestros resultados en los que los porcentajes de diferencia son muy similares, incluso en su mayoría de menor magnitud.

Teniendo en cuenta los resultados globales de las dos intensidades evaluadas, además de la totalidad de series realizadas, los coeficientes de correlación resultantes en el presente estudio oscilan entre .934 - .981 para $r$ de Pearson y .872 - .962 para $R^{2}$. Resultados similares se encontraron en los estudios comparativos de Hilmersson et al. (2015) y Squadrone et al. (2012), donde obtuvieron también altos valores en $r$ (.95 - .98) o $R^{2}$ (.978 - 0.993), respectivamente, coincidiendo en la idoneidad de los dispositivos analizados y los excelentes límites de error, en base a los resultados obtenidos. En este sentido Carlock et al. (2004) y Hori et al. (2009), definen los valores de coeficiente de correlación de Pearson > .9 como casi perfecto y 0.7-0.9 como muy altos, por lo que los datos de correlación que aporta nuestro estudio son excelentes en su mayoría (> .9). Así mismo, tanto los valores de Alpha de Cronbach (.966 - .990) como los ICC (.886 - .990) obtenidos para toda la muestra han resultado ser excelentes. En este sentido el objetivo de mejorar el rendimiento de un deportista pasa por disponer de métodos lo más exactos y precisos posible (Currell \& Jeukendrup, 2008), así como es de vital importancia que estén asociados con un equipamiento fiable, ya que de lo contrario sería complicado que el individuo pudiera hacer un correcto seguimiento de su progresión y evolución a lo largo del tiempo (Hopkins, 2000).

\section{Conclusiones}

Los excelentes resultados y el alto grado de significación en todas las correlaciones analizadas encontradas para todas las inercias y las dos intensidades analizadas, hacen de BlueBrain ${ }^{\mathrm{TM}}$ y SmartCoach $^{\mathrm{TM}}$ unas herramientas de gran calidad y validez para la medición y estimación de valores de potencia media CON y trabajo CON-EXC cuando se realizan ejercicios de fuerza en máquinas equipadas con tecnología inercial flywheel.

Aunque son resultados preliminares y exploratorios, parece que durante ejercicio a la máxima intensidad, a mayor inercia utilizada se alcanzan valores más bajos de potencia media (CON) y se obtienen resultados más elevados de trabajo (CON-EXC). Del mismo modo, a medida que se aumenta la inercia de entrenamiento, disminuye la diferencia (\%) en el registro de valores entre ambos dispositivos, estando ligeramente por encima los valores obtenidos con BB. Las inercias 0.0625 y $0.075 \mathrm{~kg}^{*} \mathrm{~m}^{2}$ muestran los valores más altos en $R^{2}$ y $r$ de Pearson para el total de series llevadas a cabo en el estudio.

Estas herramientas (BB y SC) pueden ayudar en el proceso de seguimiento, control y evolución de la progresión del deportista y a obtener un mejor ajuste de las cargas a diferentes intensidades. Del mismo modo, pueden tener otros beneficios como: complementariedad con otros elementos y dispositivos de entrenamiento; ayuda en la regulación del esfuerzo; puede ser un elemento que incentive la motivación del deportista y facilite el proceso de comunicación con el entrenador. En el terreno de la rehabilitación y prevención de lesiones, el poder realizar este tipo de mediciones permite dosificar de forma más segura y precisa la carga de entrenamiento, ya que no es necesario el uso de cargas máximas para evaluar la fuerza, reduciendo con ello pérdidas de 
tiempo y posibles riesgos lesivos.

Por último, como posibles limitaciones del estudio, podríamos destacar las dificultades para encontrar una muestra más numerosa que cumpla los criterios mínimos establecidos; la necesidad de ajustar matemáticamente las inercias iniciales de SC y por último, la complejidad para encontrar y realizar comparaciones con estudios previos, debido principalmente a que BB es un sistema de reciente implantación.

\section{Agradecimientos}

Agradecemos el apoyo logístico y colaboración de nHANCE ${ }^{\mathrm{TM}}$ al habernos permitido utilizar el dispositivo BlueBrain ${ }^{\mathrm{TM}}$ para el desarrollo del presente estudio.

\section{Referencias}

Askling, C., Karlsson, J. \& Thorstensson, A. (2003). Hamstring injury occurrence in elite soccer players after preseason strength training with eccentric overload. Scand J Med Sci Sports, 13(4):244-50.

Atkinson, G. \& Nevill, A.M. (1998). Statistical methods for assessing measurement error (reliability) in variables relevant to sports medicine. Sports Med, 26:217-38.

Carlock, J.M., Smith, S.L., Hartman, M.J., Morris, R.T., Ciroslan, D.A., Pierce, K.C.,....Stone, M.H.(2004). The relationship between vertical jump power estimates and weightlifting ability: a field-test approach. J Strength Cond Res, 18:534-9.

Cronin, J.B. \& Hansen, K.T. (2005). Strength and power predictors of sports speed. J Strength Cond Res, 19(2):349-57.

Cuenca-Fernández, F., López-Contreras, G. \& Arellano, R. (2015). Effect on swimming start performance of two types of activation protocols: lunge and YoYo squat. J Strength Cond Res, 29(3):64755.

Currell, K. \& Jeukendrup, (2008).A.E. Validity, reliability and sensitivity of measures of sporting performance. Sports Med, 23:297-316.

De Hoyo, M., Pozzo, M., Sañudo, B., Carrasco, L., Gonzalo-Skok, O., Domínguez-Cobo, S. \& Morán-Camacho, E. (2015). Effects of a 10-week in-season eccentric-overload training program on muscleinjury prevention and performance in junior elite soccer players. Int J Sports Physiol Perform, 10(1):46-52.

Drinkwater, D.J., Galna, B., McKenna, M.J., Hunt, P.H. \& Pyne, D.B. (2007). Validation of an optical encoder during free weight resistance movements and analysis of bench press sticking point power during fatigue. J Strength Cond Res, 21(2), 510-517.

Fernandez-Gonzalo, R., Lundberg, T.R., Alvarez-Alvarez, L. \& de Paz, J.A. (2014). Muscle damage responses and adaptations to eccentricoverload resistance exercise in men and women. Eur J Appl Physiol, 114:1075-1084.

Fernandez-Gonzalo, R., Nissemark, C., Aslund, B., Tesch, P.A. \& Sojka, P. (2014). Chronic stroke patients show early and robust improvements in muscle and functional performance in response to eccentric-overload flywheel resistance training: a pilot study. $J$ Neuroeng Rehabil, 11:150.

Ferrer Contreras, M.C. (2007). Efectos de dos métodos de entrenamiento de fuerza sobre el índice de Bosco en jugadoras de balonmano de División de Honor. Effects of two strength training methods in Bosco Index on elite handball female player. Retos. Nuevas tendencias en Educación Física, Deporte y Recreación, 11:33-36.

González-Badillo, J.J. \& Sánchez-Medina, L. (2010). Movement velocity as a measure of loading intensity in resistance training. International Journal of Sports Medicine, 31(5):347-352.

González-Badillo, J.J., Pareja-Blanco, F., Rodríguez-Rosell, D., AbadHerencia, J.L., Del Ojo-López, J.J., \& Sánchez-Medina, L. (2015). Effects of velocity-based resistance training on youung soccer players of different ages. J Strength Cond Res, 29(5):1329-38.

Hernández-Davó, J.L., Solana, R.S., Sarabia-Marín, J.M., et al. (2015). The rest interval required for power training with the load that maximized power output in the bench press throw exercise. $J$
Strength Cond Res, Sep 25. In press.

Hilmersson, M., Edvardsson, I. \& Tornberg, Å.B. (2015). Power of counter movement jumps with external load - coherence of three assessment methods. BMC Research Notes, 8:156.

Hopkins, W.G. (2000). Measures of reliability in sports medicine and science. Sports Med, 30:1-15.

Hori, N., Newton, R.U., Kawamori, N., McGuigan, M.R., Kraemer, W.J. \& Nosaka, K. (2009). Reliability of performance measurements derived from ground reaction force data during countermovement jump and the influence of sampling frequency. $J$ Strength Cond Res, 23:874-82.

Lindemann, U., Farahmand, P., Klenk, J., Blatzonis, K. \& Becker, C. (2015). Validity of linear encoder measurement of sit-to-stand performance power in older people. Physiotherapy, 101: 298-302.

Loturco, I., Artioli, G.G., Kobal, R., Gil, S. \& Franchini, E. (2014). Predicting punching acceleration from selected strength and power variables in elite karate athletes: a multiple regression analysis. $J$ Strength Cond Res, 28(7):1826-32.

Lundberg, T.R., Fernandez-Gonzalo, R. \& Tesch, P.A. (2014). Exerciseinduced AMPK activation does not interfere with muscle hypertrophy in response to resistance training in men. Journal of applied physiology, 116: 611-620.

Lundberg, T.R., Fernandez-Gonzalo, R., Gustafsson, T. \& Tesch, P.A. (2013). Aerobic exercise does not compromise muscle hypertrophy response to short-term resistance training. Journal of applied physiology, 114: 81-89.

Niewiadomski, W., Laskowska, D., G1siorowska, A., Cybulski, G., Strasz, A. \& Langfort, J. (2008). Determination and prediction of one repetition maximum (1RM): Safety considerations. Journal of human kinetics, 19: 109-120, 2008.

Norrbrand, L., Fluckey, J.D., Pozzo, M. \& Tesch, P.A. (2008). Resistance training using eccentric overload induces early adaptations in skeletal muscle size. Eur J Appl Physiol, 102: 271-281.

Norrbrand, L., Pozzo, M. \& Tesch, P.A. (2010). Flywheel resistance training calls for greater eccentric muscle activation than weight training. Eur J Appl Physiol, 110: 997-1005.

Onambele, G.L., Maganaris, C.N., Mian, O.S., Tam, E., Rejc, E., McEwan, I.M. \& Narici, M.V.(2008). Neuromuscular and balance responses to flywheel inertial versus weight training in olderpersons. J Biomech, 41: 3133-3138.

Ratamess, N.A. (2012). ACSM's foundations of strength training and conditioning. Wolters Kluwer Health/Lippincott Williams \& Wilkins.

Smilios, I., Sotiropoulos, K, Christou, M., Douda, H., Spaias, A. \& Tokmakidis, S.P. (2013). Maximum power training load determination and its effects on load-power relationship, maximum strength, and vertical jump performance. J Strength Cond Res, 27(5): 1223-1233.

Squadrone, R., Rodano, R. \& Preatoni, E. (2012). Comparison of velocity and power output data derived from an inertial based system and an optical encoder during squat lifts in a weight room setting. $J$ Sports Med Phys Fitness, 52:40-6.

Torres Luque, G., García-Martos, M., Villaverde Gutiérrez, C. \& Garatachea Vallejo, N. (2010). Papel del ejercicio físico en la prevención y tratamiento de la obesidad en adultos. The role of physical exercise in prevention and treatment of obesity in adults. Retos. Nuevas tendencias en Educación Física, Deporte y Recreación, 18:47-51.

Víquez Ulate, F., Mora Campos, A. (2011). Efectos de un programa de entrenamiento de fuerza con pesas en nadadores con Síndrome de Down. Effects of Weight Resistance Training on Swimmers with Down Syndrome. Retos. Nuevas tendencias en Educación Física, Deportey Recreación, 19:10-14.

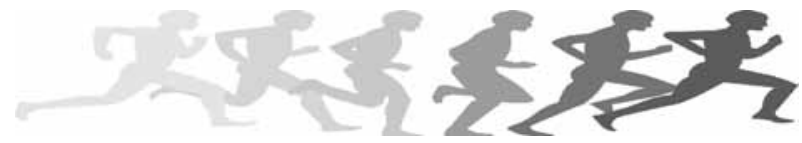

